\title{
Gimbal Device to Minimize the Effects of Off-Center Loading on Balance Pans
}

\author{
H. A. Bowman and L. B. Macurdy
}

(July 22, 1960)

\begin{abstract}
Failure to center loads on laboratory-balance pans results in swinging and vibration of the suspension upon release, both of which contribute to weighing error. As part of a study of balance design in relation to sources of uncertainty, a device has been contrived which, when installed in the suspension, locks the suspension in a gravitationally alined configuration after loading but prior to beam release. Data is presented showing significant reduction of the effects of off-centering after the device has been operated.
\end{abstract}

This is the second $[1]^{1}$ in a series of reports on studies of methods of reducing balance and weighing errors. It is directed to a reduction of uncertainties arising from imperfect centering of the load when it is placed on the pan. Such uncertainties fall into two general categories static and dynamic. Static effects are caused by variations in the effective arm length from one reading to the next which result from nonreproducibility in the mating of knives and flats and also from geometric imperfections in these members. These static errors have been reported on by previous workers $[2,3,4]$. This report is concerned with a method of reducing the dynamic errors associated with swinging and vibration of the suspension.

Ideally the suspension of a balance hangs in such a manner that the center of gravity of the suspended mass (suspension plus load) lies on a vertical axis below and at a constant distance from the effective point of support. Actually, however, it swings from this axis with an initial amplitude which is determined principally by the displacement of the center of gravity from this vertical axis. This swinging, which may be the resultant of several modes in a multiple-link suspension, causes error in observed "turning points" that is the observed limits of deflection of the beam. Upon release, ${ }^{2}$ the mechanical shock impressed on a multiple-linksuspension, as it takes on a tension load, causes it to vibrate transversely at an amplitude which is . lso principally determined by the displacement of the centers of gravity of the various links from the vertical axis. These vibrations tend to alter the relative positions of the various knives and flats, thereby changing arm length and sensitivity from one set of observations to the next.

It is difficult to evaluate, except under highly unrealistic conditions, the dynamic errors associated

${ }_{1}^{1}$ Figures in brackets indicate the literature references at the end of this paper. 2 The release of a balance beam or suspension is conventionally defined as the coming into contact of a knife edge with its plane. In this paper the term is used more generally to describe the removal of the last constraint preventing swing about the relevant knife edge. with the vibration and swinging of the suspension independently of the various static errors present. This is due to the interrelationship which exists between these two classes of errors. For example, the magnitude of a dynamic-vibration error resulting from motion of the intermediate knife is directly related to the static knife-edge parallelity-adjustment error.

A significant part of the dynamic-swing error is associated with the centripetal acceleration of the suspension rotating about the terminal knife. This is the only swinging movement one cannot eliminate altogether by locking because it is essential to the proper balance function. The maximum value of this acceleration for one suspension is $g \theta^{2}$, for small $\theta$, where $\theta$ is the peak angular amplitude of its swing: For a suspension swinging through an angle of $10^{-3}$ radians this is about $10^{-3} \mathrm{~cm} \mathrm{sec}^{-2}$, and the variability of force on the terminal knife, which this acceleration causes, is of the order of $10^{-6}$ of the total load (suspension plus useful load).

On conventional balances, to limit $\theta$ to $10^{-3}$ radians (used in the example above), care must be exercised in centering the load on the pan. The release mechanism must be operated slowly, smoothly, and with a "feeling" on the part of the operator for the instrument in order to ensure that the center of gravity of the loaded suspension is well centered below the point of support at the instant of release. To reduce the effects of indeterminate changes of ambient conditions on balance operation we are trying to devise balance parts suited to a fast, automatic, weighing system with maximum-attainable precision. A complex, slowly operating (albeit highly accurate) load-centering and balance-releasing mechanism has no place in such a system.

A feature necessary in such a system, but not discussed in detail here, is a beam with a short period. Unfortunately, however, the centripetal force, referred to above, exerts an increasingly effective disturbance on the beam (and thus on the observing system) as the frequency of the beam is increased to the order of that of the suspension. It may still be 
possible to reduce this disturbance statistically by taking "many" observations, but the time saved by virtue of a "fast" beam would thus be unacceptably dissipated. It should be noted that the period of the suspension cannot be correspondingly decreased because of the limitations imposed by the dimensions of weights and samples to be weighed.

A method of overcoming this difficulty is to ensure that $\theta$ is held within acceptably low limits. Keeping $\theta$ to a maximum value of about $10^{-5}$ radians will result in a centripetal acceleration of $10^{-7} \mathrm{~cm} \mathrm{sec}^{-2}$, which assuming a $2-\mathrm{kg}$ mass (1-kg weight and $1-\mathrm{kg}$ suspension, will result in a maximum variability of force on the terminal knife due to this cause of about $2 \times 10^{-10} \mathrm{~kg}$. Such a small disturbing force will have a trivial effect compared with other errors present, even when but "few" observations are taken on a fast-beam balance.

A practical method of restricting $\theta$ to this small value is to release the suspension with its center of gravity vertically below its center of rotation. The cause of swinging is thus removed. This center of gravity can be moved satisfactorily close to the vertical axis (through the effective point of suspension) by installing a gimbal device (fig. 1) in the suspension

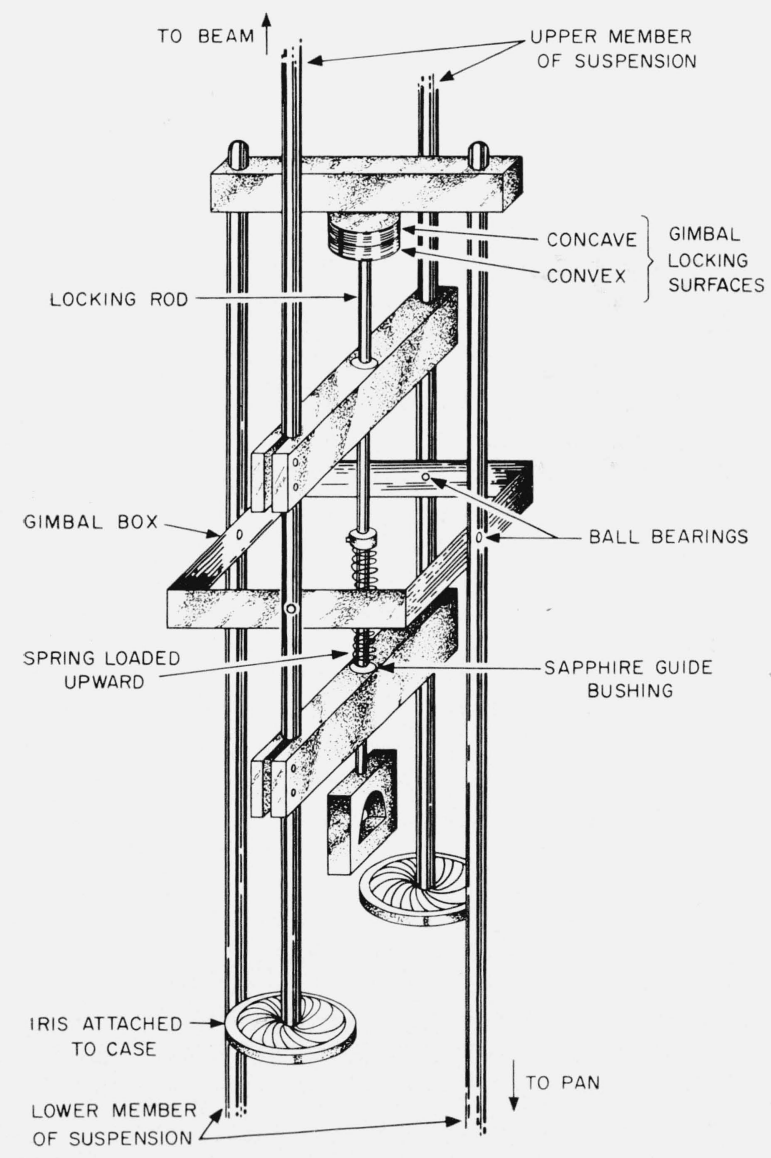

Figure 1. Diagrammatic sketch of the gimbal and its locking devices. about midway between its top and bottom. During pan loading the upper member of the suspension is clamped firmly in its normal rest position (vertically below the center of the terminal knife) by the two irislike devices attached to the balance case. The lower member of the suspension is at that time locked to the upper member (fig. 1) by the two spring loaded locking surfaces, which are spherical segments centered at the center of rotation of the gimbal. The locking rod terminating in the convex locking surface is part of the upper member. It may be moved vertically without horizontal displacement of the center of gravity of this member. After the load is placed on the pan, the locking rod is pulled vertically downward against the spring by an externally controlled gimbal release, thereby freeing the lower member to rotate about the center of rotation of the gimbal. By one of the procedures described in the following paragraph the lower member is brought to its rest position. The locking rod is then permitted to move upward until the two locking surfaces are in firm contact. The irises may now be opened, thus releasing the suspension. Since the center of gravity of each member of the suspension (including a pan with an offcenter load) lies on the vertical axis previously referred to, the tendency of the suspension to swing is minimized. Also upon release, it is effectively a single-link suspension (by virtue of the lock between the upper and lower members) so the tendency to vibrate is also minimized.

The gravitational centering of the lower member can be accomplished by lowering the locking rod for one-quarter of the pendulum period of this member. There is a tendency to overshoot the rest point when the locking surfaces first stop the swing of the lower member. The process therefore must be repeated several times. Figure 2 shows the tilting effect on the prototype suspension caused by a $30-\mathrm{g}$ load placed $2 \frac{1}{2}$ in. away from the center of the pan, before gravitational centering and after the locking surfaces had been opened 1,2,3, . .9 times. Each time the locking rod was opened during these experiments it was held open manually for roughly $0.3 \mathrm{sec}$, since the pendulum period of the lower member was 1.2 sec. With a well designed and timed automatic unlocking mechanism, it is expected that adequate centering will be achieved in 5 or 6 unlocking operations, which will require less than 10 sec. An alternative method of attaining centering with this device would be the use of a suitable damping mechanism which might permit the lower member to fall into its equilibrium position in one smooth motion. The design of a damped system has not been undertaken to date. It is probable that a satisfactory mechanism could be found, but its inherent requirements should not be underestimated. It must not only be almost free of friction, but also it must not disturb the balance through undue magnetic or electric forces, or by introducing heat or unnecessary surfaces acted upon by convective effects.

Miniature ball bearings of low-starting friction are installed in the prototype gimbal. Although the 


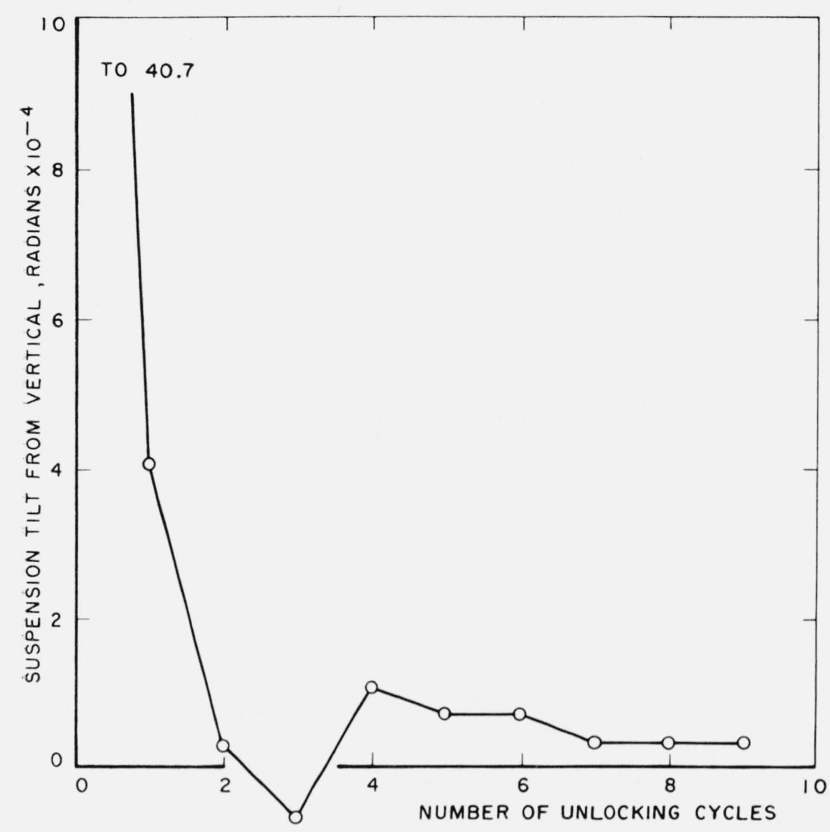

Figure 2. Plot of the residual deflection of the prototype suspension from vertical obtained by successive unlocking of the gimbal after an initial deflection due to offcenter balance pan loading.

possibility exists that knife edges and flats may yet be required in some applications, test data indicate that these bearings will satisfy all likely requirements.

The prototype suspension is released by the irises. Residual swinging of the suspension could be caused by surface forces between the irises and the rods around which they close. It is feared that these forces might be excessive for balance suspensions of the very highest order of precision. Further models of operating suspensions now under construction will be finally released by lowering needles vertically out of holes in the ends of the rods clamped by irises. The materials and surfaces of these needles and holes are chosen to minimize contact-surface forces. The irises will then be used only to steady the rods while the needles are being inserted. Thereafter the irises will be opened and play no further part in the process.

John M. Frankland provided guidance in the elastic considerations involved in background studies and in the establishment of performance requirements. The prototype model was constructed by R. A. Smith of NBS Instrument Shop under the direction of N. C. Pines, both of whom contributed many ideas embodied in the device.

\section{References}

[1] H. A. Bowman, L. B. Macurdy, A photoelectric follow-up and recording system and its application to remote observation of high precision balances, J. Research NBS 63C, 91 (1959).

[2] M. W. J. Marek, Trav. et Mem. Bur. Intern. Poids et Meas. 1 D 53 (1881).

[3] M. Thiesen, Trav. et Mem. Bur. Intern. Poids et Meas., 5 pt 2 (1886).

[4] F. A. Gould, A knife-edge balance for weighings of the highest accuracy, Proc. Phys. Soc. 62, 817 (1949).

(Paper 64C4-46) 